\title{
Oscillator with Random Mass
}

\author{
Moshe Gitterman \\ Department of Physics, Bar Ilan University, Tel Aviv, Israel \\ Email: gittem2001@yahoo.com
}

Received January 12, 2012; revised February 20, 2012; accepted March 1, 2012

\begin{abstract}
We consider an oscillator with a random mass for which the particles of the surrounding medium adhere to the oscillator for some random time after the collision (Brownian motion with adhesion). This is another form of a stochastic oscillator, different from oscillator usually studied that is subject to a random force or having random frequency or random damping. We calculated first two moments for different form of a random force, and studied different resonance phenomena (stochastic resonance, vibration resonance and "erratic" behavior) interposed between order and chaos.
\end{abstract}

Keywords: Harmonic Oscillator; Fluctuating Mass; Stability Conditions; Resonance Phenomena

\section{Introduction}

The simplest, most general and the most widely used model in physics is the harmonic oscillator. This model has been applied everywhere, from quarks to cosmology. Moreover, a person who is worried by oscillations in the stock market can relax to classical music produced by the oscillations of string instruments. The ancient Greeks already had a general idea of oscillations and used them in musical instruments. Regarding practical applications, we note the Galilean discovery of the universality of the period for small oscillations, which was used in 1602 for measuring the human pulse. Many other applications have been found in the last 400 years. Our interest here is centered on the influence of noise on the harmonic oscillator [1].

All of Newtonian mechanics is encapsulated in the basic equation, $F=m \mathrm{~d}^{2} x / \mathrm{d} t^{2}$, where the force $F$ applied to a particle of mass $m$, situated at position $x(t)$ at time $t$, causes the particle to accelerate. The goal of mechanics is to find $x(t)$, the position of the particle at any future time, given its position and velocity at some initial time $t_{0}$.

The quintessential example of a force is the one-dimensional harmonic oscillator, for which the force $F=$ $-k x$ attempts to return the oscillator to its equilibrium position. Inserting this force into Newton's equation gives

$$
m \mathrm{~d}^{2} x / \mathrm{d} t^{2}=-k x
$$

which is easily solved to yield

$$
x(t)=C \sin (w t+\alpha)
$$

where $\omega^{2}=\sqrt{k / m}$ is the angular frequency of oscillation and the two constants $C$ and $\alpha$ are determined by the initial position and initial velocity of the oscillator. Textbooks often present a generalized version of the harmonic oscillator by including a velocity-dependent frictional force, with friction constant $\gamma$,

$$
m \frac{\mathrm{d}^{2} x}{\mathrm{~d} t^{2}}=-k x-\gamma \frac{\mathrm{d} x}{\mathrm{~d} t}
$$

which also can be easily solved to yield

$$
x(t)=C \exp \left(-\frac{\gamma t}{2 m}\right) \cos \left(\omega_{1} t+\alpha\right)
$$

where the oscillator is seen to be damped exponentially by the frictional force and the angular frequency $\left(\omega_{1}^{2}=\omega^{2}-\gamma^{2} / 4\right)$ is somewhat reduced.

If the whole world were to consist solely of uncoupled harmonic oscillators, the subject of mechanics could end right here. However, most mechanical systems are much more complicated. Among the generalizations of the simple harmonic oscillator that have been considered in recent years is the stochastic oscillator, which is an oscillator that is subject to random external influences.

There are different ways of including fluctuations in the oscillator model. These may arise from internal fluctuations (thermal noise) described by

$$
\frac{\mathrm{d}^{2} x}{\mathrm{~d} t^{2}}+\frac{\gamma}{m} \frac{\mathrm{d} x}{\mathrm{~d} t}+\frac{\omega^{2}}{m} x=\xi(t)
$$

The random force $\xi(t)$ appearing on the right-hand side of the oscillator equation describes Brownian motion. An additive random force, originating from the random number of molecules of the surrounding medium that collide with the Brownian particle from opposite sides, results in random zigzag motion. There are many books 
describing different aspects and many applications of Brownian motion [2]. External fluctuations have a different origin, connected with random changes of the oscillator parameters manifested as random frequency and random damping. The former is described by the following equation (with $m=1$ ),

$$
\frac{\mathrm{d}^{2} x}{\mathrm{~d} t^{2}}+\gamma \frac{\mathrm{d} x}{\mathrm{~d} t}+\omega^{2}[1+\xi(t)] x=0
$$

The many applications of this model include different fields in physics, such as wave propagation in a random medium [3], spin precession in a random external field [4], turbulent flow on the ocean surface [5], and as well as in biology (population dynamics [6]), in economics (stock market prices [7]) and so on. The case of random damping is described by the equation

$$
\frac{\mathrm{d}^{2} x}{\mathrm{~d} t^{2}}+\gamma[1+\xi(t)] \frac{\mathrm{d} x}{\mathrm{~d} t}+\omega^{2} x=0
$$

This equation was first used [8] to analyze water waves influenced by a turbulent wind field. However, this equation, with the coordinate $x$ and time $t$ replaced by the order parameter and coordinate, respectively, transforms into the Ginzburg-Landau equation with a convective term which describes phase transitions in a moving system [9]. There are many problems in which the particle advected by the mean flow passes through the region under study, including phase transitions under shear [10], open flows of liquids [11], dendritic growth [12], chemical waves [13], motion of vortices [14], etc.

In this article, we discuss still another possibility for introducing randomness in the oscillator equation, namely, by introducing a random mass [15-19], which is described by the following equation

$$
[1+\xi(t)] \frac{\mathrm{d}^{2} x}{\mathrm{~d} t^{2}}+\gamma \frac{\mathrm{d} x}{\mathrm{~d} t}+\omega^{2} x=0
$$

There are many situations in chemical and biological solutions in which the surrounding medium contains molecules which are capable not only of colliding with the Brownian particle, but also adhering to it. A multiplicative random force arises from the adhesion of surrounding molecules which stick to the Brownian particle for some (random) time, thereby changing its mass. Modern applications of such a model include a nano-mechanical resonator which randomly absorbs and desorbs molecules [20]. The diffusion of clusters with randomly growing masses has also been considered [21]. There are some applications of a variable-mass oscillator [22]. The oscillator equation may contain both a multiplicative random force $\xi(t)$, as in Equation (8), and an additive random force $\eta(t)$

$$
[1+\xi(t)] \frac{\mathrm{d}^{2} x}{\mathrm{~d} t^{2}}+\gamma \frac{\mathrm{d} x}{\mathrm{~d} t}+\omega^{2} x=\varsigma(t)
$$

Equation (9) describes Brownian motion with adhesion. There are many other applications of an oscillator with a random mass [23], such as ion-ion reactions [2426], electrodeposition [27], granual flow [28-30], cosmology [31-34], film deposition [35], traffic jams [31,32], and the stock market $[38,39]$.

\section{White and Colored Noise}

In the following we will consider noise $\xi(t)$ with $\langle\xi(t)\rangle=0$ having the correlator

$$
\left\langle\xi\left(t_{1}\right) \xi\left(t_{2}\right)\right\rangle=r\left(\left|t_{1}-t_{2}\right|\right)
$$

Two integrals characterize the fluctuations: the strength of the noise $D$

$$
D=\int_{0}^{\infty}\langle\xi(t) \xi(t+z)\rangle \mathrm{d} z
$$

and the correlation time $\lambda$,

$$
\lambda=\frac{1}{D} \int_{0}^{\infty}\langle\xi(t) \xi(t+z)\rangle z \mathrm{~d} z
$$

\subsection{White Noise}

Traditionally one considers two different forms of noise, white and colored noise. For white noise, the function $r\left|t_{1}-t_{2}\right|$ has the form of a delta-function,

$$
\left\langle\xi\left(t_{1}\right) \xi\left(t_{2}\right)\right\rangle=D \delta\left(t_{1}-t_{2}\right)
$$

The name "white" noise comes from the fact that the Fourier transform of (13) is "white", being constant without any characteristic frequency. Equation (13) means that noises $\xi\left(t_{1}\right)$ and $\xi\left(t_{2}\right)$ are statistically independent, no matter how close $t_{1}$ and $t_{2}$ are. This extreme assumption, which leads to a non-physical infinite value of $\left\langle\xi^{2}(t)\right\rangle$ in (13), means that the correlation time $\tau$ is not zero, as assumed in (13), but smaller than all other characteristic times in the problem. It is clear that for our problem, the noise in (8) cannot be white since a large negative noise, $\xi(t) \ll 0$, implies a negative mass of the oscillator.

\subsection{Colored Noise}

All non-white sources of noise are called colored noise. We consider a special type of noise, the so-called dichotomous noise (random telegraph signal), which randomly jumps between two different values, either $\pm \sigma$ (symmetric dichotomous noise) or $A$ and $-B$ (asymmetric di- chotomous noise), which are characterized by the Orn- stein-Uhlenbeck correlation function. For the symmetric noise, one has the following form

$$
\left\langle\xi\left(t_{1}\right) \xi\left(t_{2}\right)\right\rangle=\frac{\sigma^{2}}{\lambda} \exp \left[-\lambda\left|t_{1}-t_{2}\right|\right]
$$


White noise (13) is defined by its strength $D$ while the Ornstein-Uhlenbeck noise is characterized by two parameters, $\sigma^{2}$ and $\lambda$. The transition from the OrnsteinUhlenbeck noise (14) to white noise (13) occurs in the limit $\sigma^{2} \rightarrow \infty$ and $\lambda \rightarrow \infty$, with $\sigma^{2} / \lambda=D$ in (14).

Returning to our Equation (8) and multiplying it by $1-\xi(t)$, one obtains

$$
\left(1-\sigma^{2}\right) \frac{\mathrm{d}^{2} x}{\mathrm{~d} t^{2}}+(1-\xi(t))\left(\gamma \frac{\mathrm{d}}{\mathrm{d} t}+\omega^{2}\right) x=0
$$

Since the oscillator mass is positive, the condition $\sigma^{2}<1$ should be satisfied in studies of Equation (15). For the asymptotic case of small oscillations of the mass, $\sigma^{2} \ll 1$, Equation (15) can be rewritten as

$$
\frac{\mathrm{d}^{2} x}{\mathrm{~d} t^{2}}+\gamma \frac{\mathrm{d}}{\mathrm{d} t}+\omega^{2} x=\xi(t)\left(\gamma \frac{\mathrm{d}}{\mathrm{d} t}+\omega^{2}\right) x
$$

Therefore, the small dichotomous fluctuations of mass are equivalent to simultaneous fluctuations of the frequency and the damping coefficient.

For asymmetric dichotomous noise, it is convenient to replace $\xi(t)$ in Equation (8) by a positive random force $\xi^{2}(t)$, which corresponds to the fact that the mass of the Brownian particle can only increase due to the adhesion of the molecules of the surrounding medium,

$$
\left[1+\xi^{2}(t)\right] \frac{\mathrm{d}^{2} x}{\mathrm{~d} t^{2}}+\gamma \frac{\mathrm{d} x}{\mathrm{~d} t}+\omega^{2} x=\varsigma(t)
$$

The quadratic noise $\xi^{2}(t)$ can be written as

$$
\xi^{2}(t)=\sigma^{2}+\Delta \xi
$$

with $\sigma^{2}=A B$ and $\Delta=A-B$. Indeed, for $\xi=A$, one obtains $\xi^{2}=A B+(A-B) A=A^{2}$, and for $\xi=-B$, $\xi^{2}=B^{2}$

Then, Equation (17) takes the following form,

$$
\left[1+\sigma^{2}+\Delta \xi(t)\right] \frac{\mathrm{d}^{2} x}{\mathrm{~d} t^{2}}+\gamma \frac{\mathrm{d} x}{\mathrm{~d} t}+\omega^{2} x=\varsigma(t)
$$

Multiplying Equation (19) with $\varsigma=0$ by $1+\sigma^{2}-\Delta \xi(t)$, one obtains

$$
R \frac{\mathrm{d}^{2} x}{\mathrm{~d} t^{2}}+\left(1+\sigma^{2}\right)\left(\gamma \frac{\mathrm{d} x}{\mathrm{~d} t}+\omega^{2} x\right)=\Delta \xi\left(\gamma \frac{\mathrm{d} x}{\mathrm{~d} t}+\omega^{2} x\right)
$$

where $R=\left(1+\sigma^{2}\right)^{2}-\Delta^{2} \xi^{2}$.

In the next two Sections we calculate two first moments, $\langle x\rangle$ and $\left\langle x^{2}\right\rangle$ for Equations (15) and (19) To split the correlations, we use the well-known ShapiroLoginov procedure [40] which yields for exponentially correlated noise (14),

$$
\frac{\mathrm{d}}{\mathrm{d} t}\langle\xi g\rangle=\left\langle\xi \frac{\mathrm{d} g}{\mathrm{~d} t}\right\rangle-\lambda\langle\xi g\rangle
$$

or

$$
\left\langle\xi \frac{\mathrm{d} g}{\mathrm{~d} t}\right\rangle=\left(\frac{\mathrm{d}}{\mathrm{d} t}+\lambda\right)\langle\xi g\rangle
$$

and

$$
\left\langle\xi \frac{\mathrm{d}^{n} g}{\mathrm{~d} t^{n}}\right\rangle=\left(\frac{\mathrm{d}}{\mathrm{d} t}+\lambda\right)^{n}\langle\xi g\rangle
$$

If $\mathrm{d} g / \mathrm{d} t=A+\xi$, Equation (21) becomes

$$
\frac{\mathrm{d}}{\mathrm{d} t}\langle\xi g\rangle=\left\langle\xi^{2}\right\rangle-\lambda\langle\xi g\rangle
$$

and for white noise $\left(\xi^{2} \rightarrow \infty\right.$ and $\lambda \rightarrow \infty$ with $\left.\left\langle\xi^{2}\right\rangle / \lambda=D\right)$, one gets

$$
\langle\xi g\rangle=D
$$

\section{First Moments}

\subsection{White Noise}

Equation (8) can be rewritten in the following form

$$
\frac{\mathrm{d}^{2} x}{\mathrm{~d} t^{2}}+\gamma \frac{\mathrm{d} x}{\mathrm{~d} t}+\omega^{2} x=-\xi \frac{\mathrm{d}^{2} x}{\mathrm{~d} t^{2}}
$$

Based on linear response theory, the output $x(t)$ of the system to the input $-\xi \mathrm{d}^{2} x / \mathrm{d} t^{2}$ is

$$
\begin{aligned}
& x(t) \\
& =\frac{1}{\omega_{1}} \int_{0}^{t} \exp \left[-\frac{\gamma}{2}(t-u)\right] \sin \left[\omega_{1}(t-u)\right]\left(-\xi \frac{\mathrm{d}^{2} x(u)}{\mathrm{d} t^{2}}\right) \mathrm{d} u
\end{aligned}
$$

where $\omega_{1}=\sqrt{\omega^{2}-\gamma^{2} / 4}$. Finding $\mathrm{d}^{2} x / \mathrm{d} t^{2}$ from Equation (27), inserting it into Equation (26) and using the well-known formula for splitting the correlations,

$$
\left\langle\xi(t) \xi\left(t_{1}\right) \frac{\mathrm{d}^{2} x}{\mathrm{~d} t}\left(t_{1}\right)\right\rangle=\left\langle\xi(t) \xi\left(t_{1}\right)\right\rangle\left\langle\frac{\mathrm{d}^{2} x}{\mathrm{~d} t}\left(t_{1}\right)\right\rangle
$$

one obtains for white noise,

$$
(1-\gamma D) \frac{\mathrm{d}^{2}}{\mathrm{~d} t^{2}}\langle x\rangle+\gamma \frac{\mathrm{d}}{\mathrm{d} t}\langle x\rangle+\omega^{2}\langle x\rangle=0
$$

which means the renormalization of oscillator's mass.

\subsection{Symmetric Dichotomous Noise}

Averaging Equation (15) over an ensemble of random functions $\xi(t)$ and using Equation (22) with $g=x$ leads to

$$
\begin{aligned}
& \left(1-\sigma^{2}\right) \frac{\mathrm{d}^{2}\langle x\rangle}{\mathrm{d} t^{2}}+\left(\gamma \frac{\mathrm{d}}{\mathrm{d} t}+\omega^{2}\right)\langle x\rangle \\
& -\left(\gamma \frac{\mathrm{d}}{\mathrm{d} t}+\gamma \lambda+\omega^{2}\right)\langle\xi x\rangle=-\varepsilon
\end{aligned}
$$

where we assume white-noise correlations of noises 
$\xi(t)$ and $\varsigma(t)$, that is, $\left\langle\xi(t) \varsigma\left(t_{1}\right)\right\rangle=\varepsilon \delta\left(t-t_{1}\right)$.

The new function $\langle\xi x\rangle$ enters Equation (30). One can obtain a second equation for the two functions $\langle x\rangle$ and $\langle\xi x\rangle$ by multiplying Equation (15) by $\xi(t)$ and averaging, using again Equation (22) with $g=x$ and $g=\mathrm{d} x / \mathrm{d} t$,

$$
\begin{aligned}
& {\left[\left(1-\sigma^{2}\right)\left(\frac{\mathrm{d}}{\mathrm{d} t}+\lambda\right)^{2}+\gamma \frac{\mathrm{d}}{\mathrm{d} t}+\gamma \lambda+\omega^{2}\right]\langle\xi x\rangle} \\
& -\sigma^{2}\left(\gamma \frac{\mathrm{d}}{\mathrm{d} t}+\omega^{2}\right)\langle x\rangle=0
\end{aligned}
$$

The use of dichotomous noise offers a major advantage over other types of colored noise by terminating an infinite set of higher-order correlations, using the fact that $\left\langle\xi(t)^{2}\right\rangle=\sigma^{2}$. Eliminating $\langle\xi x\rangle$ from Equations (30) and (31), one obtains the following cumbersome equation for the first moment $\langle x(t)\rangle$

$$
\begin{aligned}
& \left(1-\sigma^{2}\right) \frac{\mathrm{d}^{4}\langle x\rangle}{\mathrm{d} t^{4}}+\left[2 \gamma+2 \lambda\left(1-\sigma^{2}\right)\right] \frac{\mathrm{d}^{3}\langle x\rangle}{\mathrm{d} t^{3}} \\
& +\left[2 \omega^{2}+\gamma^{2}+3 \gamma \lambda+\lambda^{2}\left(1-\sigma^{2}\right)\right] \frac{\mathrm{d}^{2}\langle x\rangle}{\mathrm{d} t^{2}} \\
& +(\lambda+\gamma)\left(\gamma \lambda+2 \omega^{2}\right) \frac{\mathrm{d}\langle x\rangle}{\mathrm{d} t} \\
& +\omega^{2}\left[\omega^{2}+\lambda(\lambda+\gamma)\right]\langle x\rangle \\
& =-\varepsilon\left[\lambda^{2}\left(1-\sigma^{2}\right)+\gamma \lambda+\omega^{2}\right]
\end{aligned}
$$

\subsection{Asymmetric Dichotomous Noise}

Starting from Equation (19) with $\varsigma=0$, using Equation (23) with $n=2$, and averaging over noise yields

$$
\begin{aligned}
& {\left[\left(1+\sigma^{2}\right) \frac{\mathrm{d}^{2}}{\mathrm{~d} t^{2}}+\gamma \frac{\mathrm{d}}{\mathrm{d} t}+\omega^{2}\right]\langle x\rangle} \\
& +\Delta\left(\frac{\mathrm{d}}{\mathrm{d} t}+\lambda\right)^{2}\langle\xi x\rangle=0
\end{aligned}
$$

A second equation for the two functions $\langle x\rangle$ and $\langle\xi x\rangle$ can be obtained from Equation (20) by using Equation (23) (with $n=2$ and $n=1$ ),

$$
\begin{aligned}
& {\left[R \frac{\mathrm{d}^{2}}{\mathrm{~d} t^{2}}\langle x\rangle+\left(1+\sigma^{2}\right)\left(\gamma \frac{\mathrm{d}}{\mathrm{d} t}+\omega^{2}\right)\right]\langle x\rangle} \\
& -\left[\Delta^{3}\left(\frac{\mathrm{d}}{\mathrm{d} t}+\lambda\right)^{2}+\Delta \gamma\left(\frac{\mathrm{d}}{\mathrm{d} t}+\lambda\right)+\Delta \omega^{2}\right]\langle\xi x\rangle=0
\end{aligned}
$$

Excluding the correlator $\langle\xi x\rangle$ from Equations (33) and (34), one obtains a following fourth-order differential equation for $\langle x\rangle$

$$
\begin{aligned}
& {\left[\left(1+\sigma^{2}\right)^{2}+\Delta^{2}\right] \frac{\mathrm{d}^{4}}{\mathrm{~d} t^{4}}\langle x\rangle} \\
& +\left[\Delta^{2}(\gamma+2 \lambda)+2 \lambda\left(1+\sigma^{2}\right)+2 \gamma\left(1+\sigma^{2}\right)\right] \frac{\mathrm{d}^{3}}{\mathrm{~d} t^{3}}\langle x\rangle \\
& +\left[\Delta^{2}(\lambda+\gamma)+\left(1+\sigma^{2}\right)\left(2 \omega^{2}+\lambda+3 \gamma\right)+\omega^{2} \Delta^{2}+\gamma^{2}\right] \frac{\mathrm{d}^{2}}{\mathrm{~d} t^{2}}\langle x\rangle \\
& +\left[\lambda\left(1+\sigma^{2}+\Delta^{2}\right)+\gamma\right]\left(2 \omega^{2}+\lambda \gamma\right) \frac{\mathrm{d}}{\mathrm{d} t}\langle x\rangle \\
& +\omega^{2}\left[\omega^{2}+\lambda \gamma+\lambda^{2}\left(1+\sigma^{2}+\Delta^{2}\right)\right]\langle x\rangle=0
\end{aligned}
$$

\section{Second Moments}

We restrict ourselves to the case of symmetric dichotomous noise. One can rewrite the second-order differential Equation (9) as an equivalent system of two first-order differential equations

$$
\frac{\mathrm{d} x}{\mathrm{~d} t}=y ; \quad \frac{\mathrm{d} y}{\mathrm{~d} t}+\xi \frac{\mathrm{d} y}{\mathrm{~d} t}+\gamma y+\omega^{2} x=\varsigma
$$

Multiplying the first of Equation (36) by $2 x$ and the second by $2 y$ yields

$\frac{\mathrm{d}}{\mathrm{d} t} x^{2}=2 x y ; \frac{\mathrm{d}}{\mathrm{d} t} y^{2}+2 \xi y \frac{\mathrm{d} y}{\mathrm{~d} t}+2 \gamma y^{2}+2 \omega^{2} x y=2 \varsigma y$

Averaging this equation by using (25) yields

$\frac{\mathrm{d}}{\mathrm{d} t}\left\langle x^{2}\right\rangle=2\langle x y\rangle$

$\left(\frac{\mathrm{d}}{\mathrm{d} t}+2 \gamma\right)\left\langle y^{2}\right\rangle+\left(\frac{\mathrm{d}}{\mathrm{d} t}+\lambda\right)\left\langle\xi y^{2}\right\rangle+\omega^{2} \frac{\mathrm{d}\left\langle x^{2}\right\rangle}{\mathrm{d} t}=2 D_{1}$

In deriving (38), we assumed that $\varsigma(t)$ is white noise with the correlator

$$
\left\langle\varsigma\left(t_{1}\right) \varsigma\left(t_{2}\right)\right\rangle=D_{1} \delta\left(t_{2}-t_{1}\right)
$$

Analogously, multiplying Equations (36) by $y$ and $x$, respectively, summing and averaging the sum leads to

$$
\frac{\mathrm{d}}{\mathrm{d} t} x y=y^{2}-\xi x \frac{\mathrm{d} y}{\mathrm{~d} t}-\gamma x y-\omega^{2} x^{2}+\varsigma x
$$

which yields after averaging

$$
\begin{aligned}
& \frac{\mathrm{d}}{\mathrm{d} t}\langle x y\rangle \\
& =\left\langle y^{2}\right\rangle-\left(\frac{\mathrm{d}}{\mathrm{d} t}+\lambda\right)\langle\xi x y\rangle+\left\langle\xi y^{2}\right\rangle-\gamma\langle x y\rangle-\omega^{2}\left\langle x^{2}\right\rangle
\end{aligned}
$$

Equations (38) and (41) contain new correlators $\left\langle\xi y^{2}\right\rangle$ and $\langle\xi x y\rangle$. One can calculate these and the analogous correlator $\left\langle\xi x^{2}\right\rangle$ by multiplying Equations (36) and (40) by $2 \xi x, 2 \xi y$ and $\xi$, respectively, and averaging, 


$$
\begin{gathered}
\left(\frac{\mathrm{d}}{\mathrm{d} t}+\lambda\right)\left\langle\xi x^{2}\right\rangle=2\langle\xi x y\rangle \\
\left(\frac{\mathrm{d}}{\mathrm{d} t}+\lambda+2 \gamma\right)\left\langle\xi y^{2}\right\rangle+\sigma^{2} \frac{\mathrm{d}}{\mathrm{d} t}\left\langle y^{2}\right\rangle+2 \omega^{2}\langle\xi x y\rangle=0 \\
\left(\frac{\mathrm{d}}{\mathrm{d} t}+\lambda+\gamma\right)\langle\xi x y\rangle \\
=\left\langle\xi y^{2}\right\rangle-\sigma^{2} \frac{\mathrm{d}}{\mathrm{d} t}\langle x y\rangle+\sigma^{2}\left\langle y^{2}\right\rangle-\omega^{2}\left\langle\xi x^{2}\right\rangle
\end{gathered}
$$

In the case of dichotomous noise, we split the higherorder correlators into lower-order correlators by using $\xi^{2}=\sigma^{2}$, so that, for example, $\left\langle\xi^{2} y^{2}\right\rangle=\sigma^{2}\left\langle y^{2}\right\rangle$. By this means we obtain six equations, (38) and (40) - (44), for the six variables $\left\langle x^{2}\right\rangle,\left\langle y^{2}\right\rangle,\langle x y\rangle,\left\langle\xi x^{2}\right\rangle,\left\langle\xi y^{2}\right\rangle$, and $\langle\xi x y\rangle$. We will not write down the cumbersome dynamic equations for the second moments, which can be easily obtained from this system of differential equations, but shall restrict our attention to the limiting case of white noise which gives

$$
\left\langle x^{2}\right\rangle=\frac{D_{1}}{\gamma \omega^{2}}
$$

This result coincides with the well-known result for pure Brownian motion. The independence of the stationary results on the mass fluctuation is due to the fact that the multiplicative random force appears in Equation (8) in front of the higher derivative. It is remarkable that these results are significantly different from the stationary second moments for the random frequency (Equation (6)) and random damping (Equation (7)), which are, respectively,

$$
\left\langle x^{2}\right\rangle=\frac{D_{1}}{2 \omega^{2}\left(\gamma-D \omega^{2}\right)}:\left\langle x^{2}\right\rangle=\frac{D_{1}}{\gamma \omega^{2}(1-2 \gamma D)}
$$

showing the "energetic" instability [41].

It turns out that, in the presence of dichotomous oscillator mass fluctuations, the stationary second moment $\left\langle x^{2}\right\rangle$, in contrast to its white noise form (45), may lead to instability, $\left\langle x^{2}\right\rangle<0$.

\subsection{Correlation Function}

The correlation function can be found along the same lines as was done for the second moment by multiplying Equations (36) by $x\left(t_{1}\right)$ and averaging the resulting equations, which gives

$$
\begin{gathered}
\frac{\mathrm{d}}{\mathrm{d} t}\left\langle x\left(t_{1}\right) x(t)\right\rangle=\left\langle x\left(t_{1}\right) y(t)\right\rangle \\
\frac{\mathrm{d}}{\mathrm{d} t}\left\langle x\left(t_{1}\right) y(t)\right\rangle=-\left\langle\xi(t) \frac{\mathrm{d}}{\mathrm{d} t}\left\langle x\left(t_{1}\right) y(t)\right\rangle\right\rangle \\
-\gamma\left\langle x\left(t_{1}\right) y(t)\right\rangle-\omega^{2}\left\langle x\left(t_{1}\right) x(t)\right\rangle
\end{gathered}
$$

The new correlator $\left\langle\xi(t)\left\langle x\left(t_{1}\right) y(t)\right\rangle\right\rangle$ can be found by using Equation (28) leading to

$$
\begin{aligned}
& \left(\frac{\mathrm{d}}{\mathrm{d} t}+\lambda\right)\left\langle\xi(t) x\left(t_{1}\right) x(t)\right\rangle=\left\langle\xi(t) x\left(t_{1}\right) y(t)\right\rangle \\
& \left(\frac{\mathrm{d}}{\mathrm{d} t}+\lambda\right)^{2}\left\langle\xi(t) x\left(t_{1}\right) x(t)\right\rangle+\sigma^{2} \frac{\mathrm{d}}{\mathrm{d} t}\left\langle x\left(t_{1}\right) y(t)\right\rangle \\
& +\gamma\left\langle\xi(t) x\left(t_{1}\right) y(t)\right\rangle+\omega^{2}\left\langle\xi(t) x\left(t_{1}\right) x(t)\right\rangle=0
\end{aligned}
$$

From Equations (47) and (48), one can find the fourthorder differential equation for the correlation function $\left\langle x\left(t_{1}\right) x(t)\right\rangle$, which, due to the linearity of this equation, coincides with Equation (32) for the first moment.

For dichotomous noise, the correlation function shows a non-monotonic dependence on both the noise strength $\sigma^{2}$ and the inverse correlation time $\lambda^{-1}$.

\subsection{Polynomial Dichotomous Noise}

Previously we treated linear and quadratic dichotomous fluctuations of the oscillator mass. Here we consider the most general case of polynomial dichotomous noise [42], which transforms the oscillator equation to the following form

$$
\left[1+\sum_{k=1}^{n} a_{k} \xi^{k}(t)\right] \frac{\mathrm{d}^{2} x}{\mathrm{~d} t^{2}}+\gamma \frac{\mathrm{d} x}{\mathrm{~d} t}+\omega^{2} x=\varsigma(t)
$$

where $\varsigma(t)$ is white noise

$$
\left\langle\varsigma\left(t_{1}\right) \varsigma\left(t_{2}\right)\right\rangle=D_{1} \delta\left(t_{1}-t_{2}\right)
$$

For asymmetric dichotomous noise $\xi(t)$, one gets [42]

$$
\left\langle\xi^{k}(t) g(t)\right\rangle=b_{k}\langle\xi(t) g(t)\rangle+c_{k}\langle g(t)\rangle
$$

where

$$
b_{k}=\frac{A^{k}-(-B)^{k}}{A+B} ; \quad c_{k}=\frac{B A^{k}+A(-B)^{k}}{A+B}
$$

It is easy to check that for $k=2$ Equation (51) reduces to Equation (18), after multiplying the latter equation by $g(t)$ and averaging. Analogous to the previous analysis, Equation (49) can be rewritten as two first order differential equations,

$$
\begin{aligned}
& \frac{\mathrm{d} x}{\mathrm{~d} t}=y ; \\
& \frac{\mathrm{d} y}{\mathrm{~d} t}=-\frac{\mathrm{d} y}{\mathrm{~d} t}-\sum_{k=1}^{n} a_{k} \xi^{k}(t) \frac{\mathrm{d} y}{\mathrm{~d} t}-\gamma y-\omega^{2} x+\varsigma(t)
\end{aligned}
$$

Multiplying the first equation by $2 x$ and the second by $2 y$ and averaging one gets after using Equations (51) and (23) 


$$
\begin{aligned}
& \frac{\mathrm{d}\left\langle x^{2}\right\rangle}{\mathrm{d} t}=2\langle x y\rangle ; \\
& \frac{\mathrm{d}\left\langle y^{2}\right\rangle}{\mathrm{d} t}=-\frac{\mathrm{d}\left\langle y^{2}\right\rangle}{\mathrm{d} t}-_{k=1}^{n} a_{k}\left[b_{k}\left(\frac{\mathrm{d}}{\mathrm{d} t}+\lambda\right)\left\langle\xi(t) y^{2}\right\rangle+c_{k} \frac{\mathrm{d}\left\langle y^{2}\right\rangle}{\mathrm{d} t}\right]-2 \gamma\left\langle y^{2}\right\rangle-2 \omega^{2}\langle x y\rangle+2 D_{1}
\end{aligned}
$$

Equation (54) contains the new correlator $\left\langle\xi y^{2}\right\rangle$. One can calculate this correlator by multiplying Equa-

$$
\begin{aligned}
& \left(\frac{\mathrm{d}}{\mathrm{d} t}+\lambda\right)\left\langle\xi x^{2}\right\rangle=2\langle\xi x y\rangle \\
& 2\left(\frac{\mathrm{d}}{\mathrm{d} t}+\lambda\right)\left\langle\xi y^{2}\right\rangle=-{ }_{k=1}^{n} a_{k}\left(\frac{\mathrm{d}}{\mathrm{d} t}+\lambda\right)\left\langle\xi^{k+1}(t) y^{2}\right\rangle-2 \gamma\left\langle\xi y^{2}\right\rangle-2 \omega^{2}\langle\xi x y\rangle
\end{aligned}
$$

or, by inserting (51) with $k$ replaced by $k+1$ into the last equation in Equation (55),

$$
\begin{aligned}
& 2\left(\frac{\mathrm{d}}{\mathrm{d} t}+\lambda\right)\left\langle\xi y^{2}\right\rangle \\
& =-\sum_{k=1}^{n} a_{k}\left(\frac{\mathrm{d}}{\mathrm{d} t}+\lambda\right)\left[b_{k+1}\left\langle\xi y^{2}\right\rangle+c_{k+1}\left\langle y^{2}\right\rangle\right] \\
& -2 \gamma\left\langle\xi y^{2}\right\rangle-2 \omega^{2}\langle\xi x y\rangle
\end{aligned}
$$

tions (53) by $2 \xi x$ and $2 \xi y$, which gives after averaging

which yields, after averaging and using Equation (51),

$$
2 \frac{\mathrm{d}}{\mathrm{d} t}\langle x y\rangle=2 y^{2}-\sum_{k=1}^{n} a_{k}\left[\left(\frac{\mathrm{d}}{\mathrm{d} t}+\lambda\right)\left(b_{k}\langle\xi x y\rangle+c_{k}\langle x y\rangle\right)-b_{k}\left\langle\xi y^{2}\right\rangle-c_{k}\left\langle y^{2}\right\rangle\right]-\gamma\langle x y\rangle-\omega^{2}\left\langle x^{2}\right\rangle
$$

Multiplying Equation (57) by $\xi$ and averaging, one obtains

$$
2\left(\frac{\mathrm{d}}{\mathrm{d} t}+\lambda\right)\langle\xi x y\rangle=2\left\langle\xi y^{2}\right\rangle-\sum_{k=1}^{n} a_{k}\left[\left(\frac{\mathrm{d}}{\mathrm{d} t}+\lambda\right)\left(b_{k+1}\langle\xi x y\rangle+c_{k+1}\langle x y\rangle\right)-\left(b_{k+1}\left\langle\xi y^{2}\right\rangle+c_{k+1}\left\langle y^{2}\right\rangle\right)\right]-\gamma\langle\xi x y\rangle-\omega^{2}\left\langle\xi x^{2}\right\rangle
$$

In this way we obtain six equations, (54)-(56) and (58)-(59), for the six variables $\left\langle x^{2}\right\rangle,\left\langle y^{2}\right\rangle,\langle x y\rangle$, $\left\langle\xi x^{2}\right\rangle,\left\langle\xi y^{2}\right\rangle$, and $\langle\xi x y\rangle$. We will not solve these cumbersome dynamic equations, but present only the solution for the stationary $(\mathrm{d} / \mathrm{d} t \cdots=0)$ second moment $\left\langle x^{2}\right\rangle$,

$$
\left\langle x^{2}\right\rangle=\frac{D_{1}}{\gamma \omega^{2}}\left\{\frac{S_{1} S_{4}\left[2 \omega^{2}-\lambda W-\omega^{2} \lambda\left(2+S_{3}\right) / \gamma\right]}{(U W+2 V)}+\left(2+S_{3}\right) \omega^{2}\right\}
$$

where

$$
\begin{array}{ll}
\sum_{k=1}^{n} a_{k} b_{k} \equiv S_{1} ; & \sum_{k=1}^{n} a_{k} b_{k+1} \equiv S_{2} \\
\sum_{k=1}^{n} a_{k} c_{k} \equiv S_{3} & \sum_{k=1}^{n} a_{k} c_{k+1} \equiv S_{4}
\end{array}
$$

and

$$
\begin{aligned}
& U=\frac{\lambda\left(2 \lambda+\lambda S_{2}+\gamma\right)+2 \omega^{2}}{\omega^{2} \lambda} ; \\
& V=2+S_{2}-\frac{\lambda S_{1} S_{4}}{2 \gamma} ; \\
& W=\left[\lambda\left(2+S_{2}\right)+2 \gamma+\frac{\lambda S_{1} S_{4}}{2 \gamma}\right]
\end{aligned}
$$

As one can see from Equation (60), the polynomial dichotomous fluctuations of the oscillator mass can lead to instability, $\left\langle x^{2}\right\rangle<0$, for some values of the parameters.

\section{Resonance Phenomena}

The simplest example of mechanical resonance is a harmonic oscillator subject to a periodic force, where the steady-state amplitude of the oscillator approaches infinity when the external force frequency approaches the eigenfrequency of the oscillator. This phenomenon was probably already known to the ancient Egyptians who invented the water clock, but the classical demonstration of dynamic resonance are quite recent architectural flaws 
uncovered in the US. The first was the Takoma bridge which was destroyed by the wind force at the resonance frequency, and the second was the Paramount Communication Building in New York where the winds twisted the top floors and pried windows loose from their casements.

One of greatest achievements of twentieth-century physics was establishing a deep relationship between deterministic and random phenomena. The widely studied phenomena of "deterministic chaos" and "stochastic resonance" might sound contradictory, consisting of halfdeterministic and half-random terms. However, this impression is faulty due to the close connection between determinism and randomness, although apparently different forms of behavior [43].

Here we consider a new manifestation of the resonance of an oscillator. The dynamic equation of motion of a bistable underdamped one-dimensional oscillator driven by a multiplicative random force $\alpha \xi(t)$, an additive random force $\beta \varsigma(t)$, and two periodic forces, $A \sin (\omega t)$ and $C \sin (\Omega t)$, has the following form

$$
\begin{aligned}
& \frac{\mathrm{d}^{2} x}{\mathrm{~d} t^{2}}+\gamma \frac{\mathrm{d} x}{\mathrm{~d} t}-\omega_{0}^{2} x+\alpha \xi(t) x+b x^{3} \\
& =\beta \varsigma(t)+A \sin (\omega t)+C \sin (\Omega t)
\end{aligned}
$$

The dynamic resonance mentioned above corresponds to $\gamma=b=\alpha=\beta=C=0$ and $\omega \rightarrow \omega_{0}$. Let us consider some other limiting cases of Equation (63).

1) Brownian motion ( $\left.\omega_{0}=b=\alpha=A=C=0\right)$ has been studied most widely with many applications. The equilibrium distribution comes from the balance of two contrary processes: the random force which tends to increase the velocity of the Brownian particle and the damped force which tries to stop the particle [1].

2) The double-well oscillator with additive noise ( $\alpha=A=C=0$ ) and small damping, $\gamma \ll \omega$, shows two or three peaks in the power spectrum (Fourier component of the correlation function) descriptive of fluctuation transitions between the two stable points of the potential, small intra-well vibrations and the over-thebarrier vibrations [44].

3) Stochastic resonance (SR) in overdamped $\left(\mathrm{d}^{2} x / \mathrm{d} t^{2}=\alpha=C=0\right)$ and underdamped $(\alpha=C=0)$ oscillators is a very interesting and counterintuitive phenomenon, where the noise increases a weak input signal. SR occurs in the case that a deterministic time-scale of the external periodic field is synchronized with a stochastic time-scale, determined by the Kramer transition rate over the barrier.

4) Stochastic resonance in a linear overdamped oscillator $\left(\mathrm{d}^{2} x / \mathrm{d} t^{2}=\beta=b=C=0\right)$, as distinct from the nonlinear case, allows an exact solution $[45,46]$. However, this effect occurs only when the multiplicative noise $\xi(t)$ is colored and not white.

5) Vibrational resonance $(\alpha=\beta=0)$, which occurs in a deterministic system, manifests itself in the enhancement of a weak periodic signal through a high-frequency periodic field, instead of through noise, as in the case of stochastic resonance.

6) "Erratic" behavior shows up as a "random-like" phenomenon in a simple system

$\mathrm{d}^{2} x / \mathrm{d} t^{2}=\mathrm{b}=\alpha=\beta=0$ with two incommensurate external frequencies, $\omega$ and $\Omega$.

\subsection{Stochastic Resonance}

Noise, which always plays a distractive role, appears as a constructive force, increasing the output signal as a function of noise intensity. This phenomenon was proposed as the explanation of the periodicity of the ice ages [47, 48] and has found many applications [49].

The standard definition of stochastic resonance (SR) is the non-monotonic dependence of an output signal, or some function of it, as a function of some characteristic of the noise or of the periodic signal [49]. At first glance, it appears that all three ingredients, nonlinearity, periodic forcing and random forcing, are necessary for the appearance of SR. However, it has become clear that SR is generated not only in a typical two-well system, but also in a periodic structure [50]. Moreover, SR occurs even when each of these ingredients is absent. Indeed, SR exists in linear systems when the additive noise is replaced by nonwhite multiplicative noise [46]. Deterministic chaos may induce the onset of SR instead of a random force [49]. Finally, the periodic signal may be replaced by a constant force in underdamped systems [51].

Consider the linearized Equation (19) with $\varsigma=0$ of an oscillator with random mass subject to an external periodic field,

$$
\left[1+\sigma^{2}+\Delta \xi(t)\right] \frac{\mathrm{d}^{2} x}{\mathrm{~d} t^{2}}+2 \gamma \frac{\mathrm{d} x}{\mathrm{~d} t}+\omega^{2} x=a \sin (\Omega t)
$$

Repeating the procedure leading to Equation (35), one obtains a fourth-order differential equation for $\langle x\rangle$,

$$
\begin{aligned}
& {\left[\left(1+\sigma^{2}\right)^{2}+\Delta^{2}\right] \frac{\mathrm{d}^{4}}{\mathrm{~d} t^{4}}\langle x\rangle} \\
& +\left[\Delta^{2}(\gamma+2 \lambda)+2 \lambda\left(1+\sigma^{2}\right)+2 \gamma\left(1+\sigma^{2}\right)\right] \frac{\mathrm{d}^{3}}{\mathrm{~d} t^{3}}\langle x\rangle \\
& +\left[\Delta^{2}(\lambda+\gamma)+\left(1+\sigma^{2}\right)\left(2 \omega^{2}+\lambda+3 \gamma\right)+\omega^{2} \Delta^{2}+\gamma^{2}\right] \frac{\mathrm{d}^{2}}{\mathrm{~d} t^{2}}\langle x\rangle \\
& +\left[\lambda\left(1+\sigma^{2}+\Delta^{2}\right)+\gamma\right]\left(2 \omega^{2}+\lambda \gamma\right) \frac{\mathrm{d}}{\mathrm{d} t}\langle x\rangle \\
& +\omega^{2}\left[\omega^{2}+\lambda \gamma+\lambda^{2}\left(1+\sigma^{2}+\Delta^{2}\right)\right]\langle x\rangle \\
& =\left[\omega^{2}+\left(\omega^{2}-\Omega^{2}\right)\left(1+\sigma^{2}+\Delta^{2}\right)\right] a \sin (\Omega t) \\
& +\left[\gamma+2 \lambda\left(1+\sigma^{2}+\Delta^{2}\right)\right] a \Omega \cos (\Omega t)
\end{aligned}
$$


In a similar way, one can obtain the equation for the second moment $\left\langle x^{2}\right\rangle$, associated with Equation (9), which is transformed into six equations for six variables, $\left\langle x^{2}\right\rangle,\left\langle y^{2}\right\rangle,\langle x y\rangle,\left\langle\xi x^{2}\right\rangle,\left\langle\xi y^{2}\right\rangle$ and $\langle\xi x y\rangle$, but we shall not write down these cumbersome equations.

Analogous to the cases of random frequency and random damping [47], we seek the solution of Equation (65) in the form

$$
\langle x\rangle=A \sin (\Omega t+\phi)
$$

One easily finds

$$
A=\left(\frac{f_{5}^{2}+f_{6}^{2}}{f_{7}^{2}+f_{8}^{2}}\right)^{1 / 2} ; \phi=\tan ^{-1}\left(\frac{f_{5} f_{7}+f_{6} f_{8}}{f_{5} f_{8}-f_{6} f_{7}}\right)
$$

with

$$
\begin{aligned}
f_{5}= & \left(f_{4}-f_{2} \Omega^{2}\right) a ; \quad f_{6}=\Omega f_{3} a \\
f_{7}= & \Omega^{3}\left(2 \gamma f_{2}+f_{1} f_{3}-2 \Delta^{2} \lambda \sigma^{2}\right)-\Omega\left(2 \gamma f_{4}+\omega^{2} f_{3}\right) \\
f_{8}= & \omega^{2} f_{4}-\Omega^{2}\left(\omega^{2} f_{2}+f_{1} f_{4}+2 \gamma f_{3}-\Delta^{2} \lambda^{2} \sigma^{2}\right) \\
& +\Omega^{4}\left(f_{1} f_{2}-\Delta^{2} \sigma^{2}\right) \\
f_{1} & =1+\sigma^{2} ; \quad f_{2}=1+\sigma^{2}+\Delta^{2} \\
f_{3} & =\gamma+2 \lambda f_{2} ; \quad f_{4}=\omega^{2}+\lambda\left(\gamma+\lambda f_{2}\right)
\end{aligned}
$$

One can compare Equations (66)-(68) with the equations for the first moment $\langle x\rangle$, obtained [52] for the cases of random frequency and random damping, respectively, subject to symmetric dichotomous noise, and extended afterwards $[53,54]$ to the case of asymmetric noise. All these equation are of fourth order with the same dependence on the frequency $\Omega$ of the external field but with a slightly different dependence on the parameters of the noise.

\subsection{Vibrational Resonance}

Like stochastic resonance, vibrational resonance manifests itself in the enhancement of a weak periodic signal through a high-frequency periodic field, instead of through noise as in the case of stochastic resonance. The deterministic equation of motion then has the following form,

$$
\frac{\mathrm{d}^{2} x}{\mathrm{~d} t^{2}}+\gamma \frac{\mathrm{d} x}{\mathrm{~d} t}-\omega_{0}^{2} x+b x^{3}=A \sin (\omega t)+C \sin (\Omega t)
$$

Equation (69) describes an oscillator moving in a symmetric double-well potential $V(x)=-\omega_{0}^{2} x^{2} / 2+b x^{4} / 4$ with a maximum at $x^{*}=0$ and two minima $x_{ \pm}^{*}$ with the depth $d$ of the wells,

$$
x_{ \pm}^{*}= \pm \sqrt{\frac{\omega_{0}^{2}}{b}} \quad d=\frac{\omega_{0}^{4}}{4 b}
$$

The amplitude of the output signal as a function of the amplitude $C$ of the high-frequency field has a bell shape, showing the phenomenon of vibrational resonance. For $\omega$ close to the frequency $\omega_{0}$ of the free oscillations, there are two resonance peaks, whereas for smaller $\omega$, there is only one resonance peak. These different results correspond to two different oscillatory processes, jumps between the two wells and oscillations inside one well.

Assuming that $\Omega \gg \omega$, resonance-like behavior ("vibrational resonance" [55]) manifests itself in the response of the system at the low-frequency $\omega$, which depends on the amplitude $C$ and the frequency $\Omega$ of the highfrequency signal. The latter plays a role similar to that of noise in SR. If the amplitude $C$ is larger than the barrier height $d$, the field during each half period $\pi / \Omega$ transfers the system from one potential well to the other. Moreover, the two frequencies $\omega$ and $\Omega$ are similar to the frequencies of the periodic signal and the Kramers rate of jumps between the two minima of the underdamped oscillator. Therefore, by choosing an appropriate relation between the input signal $A \sin (\omega t)$ and the amplitude $C$ of the large signal (or the strength of the noise) one can obtain a non-monotonic dependence of the output signal on the amplitude $C$ (vibration resonance) or on the noise strength (stochastic resonance). To put this another way [56], both noise in SR and the high-frequency signal in vibrational resonance change the parameters of the system response to a low-frequency signal.

Let us now pass to an approximate analytical solution of Equation (69). In accordance with the two times scales in this equation, we seek a solution of Equation (69) in the form

$$
x(t)=y(t)-\frac{C \sin (\Omega t)}{\Omega^{2}}
$$

where the first term varies significantly only over times $t$, while the second term varies much more rapidly. On substituting Equation (71) into (69), one can average over a single cycle of $\sin (\Omega t)$. Then, odd powers of $\sin (\Omega t)$ vanish upon averaging, while the $\sin ^{2}(\Omega t)$ term gives $1 / 2$. In this way, one obtains the following equation for $y(t)$,

$$
\frac{\mathrm{d}^{2} y}{\mathrm{~d} t^{2}}+\gamma \frac{\mathrm{d} y}{\mathrm{~d} t}-\left(\omega_{0}^{2}-\frac{3 b C^{2}}{2 \Omega^{4}}\right) y+b y^{3}=A \sin \omega t
$$

with

$$
\begin{aligned}
& y_{0}^{*}=0 ; y_{ \pm}^{*}= \pm \sqrt{\frac{\omega_{0}^{2}-3 b C^{2} / 2 \Omega^{4}}{b}} ; \\
& d=\frac{\left[\omega_{0}^{2}-3 b C^{2} / 2 \Omega^{4}\right]}{4 b}
\end{aligned}
$$

One can say that Equation (72) is the "coarse-grained" version (with respect to time) of Equation (69). For $3 \beta C^{2} / 2 \Omega^{4}>\omega_{0}^{2}$, the phenomenon of dynamic stabilization [57] occurs, namely, the high-frequency external 
field transforms the previously unstable position $\Psi=0$ into a stable position.

Seeking the solution of Equation (72) of the form

$$
y(t) \approx y^{*}+\Theta \sin (\omega t-\theta)
$$

and linearizing Equation (72) in $\Theta$ gives

$$
\Theta=\frac{A}{\sqrt{\left(\omega_{1}^{2}-\omega^{2}\right)^{2}+\gamma^{2} \omega^{2}}}
$$

where

$$
\omega_{1}^{2}=\frac{3 b C^{2}}{2 \Omega^{4}}-\omega_{0}^{2}+3 b\left(y^{*}\right)^{2}
$$

A resonance in the linearized Equation (72) occurs when $\omega_{1}=\omega$, which, after substituting in Equation (72), leads to the following relations between the amplitudes and frequencies of the two driving fields which produce the resonant behavior,

$$
\omega^{2}=\frac{3 b C^{2}}{2 \Omega^{4}}-\omega_{0}^{2}+\frac{3 b A^{2}}{4 \gamma^{2} \omega^{2}}
$$

In addition to the resonance phenomenon, one can study [58] the influence of the positions and depths of the potential on the vibrational resonance. Assuming that $\omega_{0}^{2}=b$, which means, according to Equation (73), that positions of minima remain fixed, let us raise the question for which value of a control parameter $C$ the ratio of the output signal $\Theta$ to the input signal $A$ is maximal. According to Equation (75), this occurs when

$S=\left(\omega_{1}^{2}-\omega^{2}\right)^{2}+\gamma^{2} \omega^{2}$ is minimal, which is determined by the condition $\mathrm{d} S / \mathrm{d} C=0$, which, using (76) with $\omega_{0}^{2}=b$, results in

$$
\begin{aligned}
& 2\left(\omega_{1}^{2}-\omega^{2}\right) \frac{\mathrm{d} \omega_{1}^{2}}{\mathrm{~d} C} \\
& =\frac{3 b C}{\Omega^{4}}\left[\frac{3 b C^{2}}{2 \Omega^{4}}-b-\omega^{2}+3 b\left(y^{*}\right)^{2}\right]=0
\end{aligned}
$$

or, for $y_{0}^{*}=0$,

$$
C^{2}=\frac{2 \Omega^{4}}{3 b}\left(b+\omega^{2}\right)
$$

and for $y_{ \pm}^{*}= \pm \sqrt{\frac{\omega_{0}^{2}}{b}-\frac{3 C^{2}}{2 \Omega^{4}}}$,

$$
C^{2}=\frac{\Omega^{4}}{3 b}\left(2 b-\omega^{2}\right)
$$

Equation (80) has real solutions for $C$ only if $2 b>\omega^{2}$.

Thus far, we considered equal values of two control parameters, $\omega_{0}^{2}=b$ changing the depths of potential and keeping the positions of minima $x_{ \pm}^{*}$ unaltered. Ana- logously, one can assume that $\omega_{0}^{4}=b$ changing thereby the distance between minima and not the potential depth. Then, one obtains, for $y_{0}^{*}=0$,

$$
C^{2}=\frac{2 \Omega^{2}}{3}\left(2+\frac{\omega^{2}}{b}\right)
$$

and for $y_{ \pm}^{*}= \pm \sqrt{\frac{\omega_{0}^{2}}{b}-\frac{3 C^{2}}{2 \Omega^{4}}}$

$$
C^{2}=\frac{2 \Omega^{2}}{3}\left(2-\frac{\omega^{2}}{b}\right)
$$

with the proviso that $2 b>\omega^{2}$.

All the above results have been obtained for an underdamped oscillator. It turns out $[59,60]$ that a similar effect also takes place for an overdamped oscillator $\left(\mathrm{d}^{2} x / \mathrm{d} t^{2}=0\right.$ in Equation (69)). The influence of the additional additive noise on the vibrational resonance, and the advantages of the vibrational resonance compared to the stochastic resonance in the detection of weak signals have been studied [61].

For an oscillator with random mass one has to add two periodic fields to Equations (15), (19), and perform the preceding analysis of Equation (69), based on dividing its solution in the two time scales (Equation (71)) followed by the linearization of Equation (72) for the slowly changing solution. The subsequent analysis of an oscillator equation with one periodic force is quite analogous to analysis of Equation (64), which describes the stochastic resonance phenomenon.

Equation (69) describes an oscillator moving in symmetric double-well potential. The vibrational resonance in the quintic oscillator with the potential of the form

$$
V(x)=\frac{1}{2} \omega_{0}^{2} x^{2}+\frac{1}{4} b x^{4}+\frac{1}{6} c x^{6}
$$

was studied in $[62,63]$. Finally, the vibrational resonance and an appearance of chaos in the Van der Pol oscillator were investigated in [64]. Because of the many applications in physics, chemistry, biology and engineering, vibrational resonance still attracts great interest, and new applications will surely be found in the future.

\section{3. "Erratic" Behavior}

One of the great achievements of twentieth-century physics was the prediction of deterministic chaos which appears in the equations without any random force [65]. Deterministic chaos means an exponential increase in time of the solutions for even the smallest change in the initial conditions. Therefore, to obtain a "deterministic" solution, one would have to specify the initial conditions to an infinite number of digits. Otherwise, the solutions of deterministic equations show chaotic behavior. Deterministic chaos occurs if the differential equations are 
nonlinear and contain at least three variables. This points to the important difference between underdamped and overdamped equations of an oscillator, since deterministic chaos may occur only in the underdamped oscillator. Here, we present an example of "erratic" behavior, which, like deterministic chaos, is drawn midway between deterministic and stochastic behavior.

Consider the simple example of an overdamped oscillator subject to two periodic fields,

$$
\frac{\mathrm{d} x}{\mathrm{~d} t}+\omega^{2} x=C_{1} \cos \left(\omega_{1} t\right)+C_{2} \cos \left(\omega_{2} t\right)
$$

We show that the solutions of this equation are "erratic", being intermediate between deterministic and chaotic solutions.

The stationary solutions of Equation (84) have the following form

$$
x(t)=\frac{C_{1}}{\omega_{1}} \sin \left(\omega_{1} t\right)+\frac{C_{2}}{\omega_{2}} \sin \left(\omega_{2} t\right)
$$

Replace the continuous time in Equation (84) by discrete times $2 \pi n / \omega_{2}$ [66]. The solution of this equation then becomes

$$
x\left(n \frac{2 \pi}{\omega_{2}}\right)=x(0)+\frac{C_{1}}{\omega_{1}} \sin \left(2 \pi n \frac{\omega_{1}}{\omega_{2}}\right)
$$

If $\omega_{1} / \omega_{2}$ is an irrational number, the sin factor in (86) will never vanish and the motion becomes "erratic". The properties of "erratic" motion can be understood from the analysis of the correlation function associated with the $n$-th and $(n+m)$-th points,

$$
\begin{aligned}
C & \left(2 \pi m \omega_{1} / \omega_{2}\right) \\
= & \lim _{N \rightarrow \infty} \frac{1}{N}_{n=0}^{N} x\left(2 \pi n \omega_{1} / \omega_{2}\right) x\left[2 \pi(n+m) \omega_{1} / \omega_{2}\right] \\
= & x^{2}(0) \\
+ & x(0)\left(C_{1} / \omega_{1}\right) \\
& \cdot \lim _{N \rightarrow \infty} \frac{1}{N}_{n=0}^{N}\left\{\sin \left(2 \pi n \omega_{1} / \omega_{2}\right)+\sin \left[2 \pi(n+m) \omega_{1} / \omega_{2}\right]\right\} \\
+ & \left(C_{1} / \omega_{1}\right)^{2} \\
\cdot & \lim _{N \rightarrow \infty} \frac{1}{N}_{n=0}^{N} \sin \left(2 \pi n \omega_{1} / \omega_{2}\right) \sin \left[2 \pi(n+m) \omega_{1} / \omega_{2}\right]
\end{aligned}
$$

Using the well-known relations between the trigonometric functions, one obtains

$$
C\left(m \frac{2 \pi \omega_{1}}{\omega_{2}}\right)=x^{2}(0)+\frac{1}{2}\left(\frac{C_{1}}{\omega_{1}}\right)^{2} \cos \left(m \frac{2 \pi \omega_{1}}{\omega_{2}}\right)
$$

The Fourier spectrum of the correlation function (88) depends on the ratio $\omega_{1} / \omega_{2}$. If this ratio is a rational number, this spectrum will contain a finite number of peaks. However, for irrational $\omega_{1} / \omega_{2}$, the spectrum becomes broadband, what is typical of deterministic chaos. However, this "erratic" behavior arises from a simple "integrable" Equation (84), which distinguishes it from deterministic chaos.

\section{Conclusion}

We considered a new type of stochastic oscillator which has a random mass. An example is Brownian motion with adhesion, where the surrounding molecules not only collide with the Brownian particle inducing a zigzag motion, but also adhere to it for a random period of time, thereby increasing the mass of the Brownian particle. The first two moments are found for dichotomous random noise. An analysis was performed of the "erratic" motion, stochastic and vibration resonances, which shows that deterministic and random phenomena are complimentary and not contradictory. Due to many applications in physics, chemistry, biology and engineering, the model of an oscillator with random mass will find many applications in the future.

\section{REFERENCES}

[1] M. Gitterman, "The Noisy Oscillator: The First Hundred Years, from Einstein until Now," World Scientific, Singapore, 2005.

[2] R. Mazo, "Brownian Motion: Fluctuations, Dynamics, and Applications," Oxford Science Publication, Oxford, 2002.

[3] A. Ishimaru, "Wave Propagation and Scattering in Random Media," John Wiley and Sons, New York, 1999.

[4] R. Kubo "A Stochastic Theory in Line Shape," In: K. E. Shuler, Ed., Stochastic Processes in Chemical Physics, Wiley, New York, 1969, pp. 101-128.

[5] O. M. Phillips, "The Dynamics of the Upper Ocean," Cambridge University Press, Cambridge, 1977.

[6] M. Turelli, "Theoretical Population Biology," Academic, New York, 1977.

[7] H. Takayasu, A.-H. Sato and M. Takayasu, "Stable Infinite Variance Fluctuations in Randomly Amplified Langevin Systems," Physical Review Letters, Vol. 79, No. 6, 1997, pp. 966-969. doi:10.1103/PhysRevLett.79.966

[8] B. West and V. Seshadri, "Model of Gravity Wave Growth Due to Fluctuations in the Air-Sea Coupling Parameter," Journal of Geophysical Research, Vol. 86, No. 5, 1981, pp. 4293-4296. doi:10.1029/JC086iC05p04293

[9] M. Gitterman, "Phase Transitions in Moving Systems," Physical Review E, Vol. 70, No. 3, 2003, Article ID: 036116. doi:10.1103/PhysRevE.70.036116

[10] A. Onuki, "Phase Transitions of Fluids in Shear Flow," Journal of Physics: Condensed Matter, Vol. 9, No. 29, 1997, pp. 6119-6159. doi:10.1088/0953-8984/9/29/001 
[11] J. M. Chomaz and A. Couairon, "Against the Wind," Physics of Fluids, Vol. 11, No. 10, 1999, pp. 2977-2984. doi:10.1063/1.870157

[12] F. Hestol and A. Libchaber, "Unidirectional Crystal Growth and Crystal Anisotropy," Physica Scripta, Vol. 1985, No. 9, 1985, pp. 126-129.

[13] A. Saul and K. Showalter, "Propagating Reaction Diffusion Fronts," In: R. J. Field and M. Burger, Eds., Oscillations and Traveling Waves in Chemical Systems, Wiley, New York, 1985, pp. 419-439.

[14] M. Gitterrman, B. Ya. Shapiro and I. Shapiro, "Phase Transitions in Vortex Matter Driven by Bias Current," Physical Review B, Vol. 65, No. 17, 2002, Article ID: 174510. doi:10.1103/PhysRevB.65.174510

[15] M. Gitterman, "New Stochastic Equation for a Harmonic Oscillator: Brownian Motion with Adhesion," Journal of Physics C: Conference Series, Vol. 248, No. 1, 2010, Article ID: 012049.

[16] M. Gitterman "New Type of Brownian Motion," Journal of Statistical Physics, Vol. 146, No. 1, 2010, pp. 239-243.

[17] M. Gitterman and V. I. Kljatskin, "Brownian Motion with Adhesion: Harmonic Oscillator with Fluctuating Mass," Physical Review E, Vol. 81, No. 5, 2010, Article ID: 051139.

[18] M. Gitterman and I. Shapiro, "Stochastic Resonance in a Harmonic Oscillator with Random Mass Subject to Asymmetric Dichotomous Noise" Journal of Statistical Physics, Vol. 144, No. 1, 2011, pp. 139-149.

[19] M. Gitterman "Harmonic Oscillator with Fluctuating Mass," Journal of Modern Physics, Vol. 2, 2010, pp. 1136-1140.

[20] J. Portman, M. Khasin, S. W. Shaw and M. I. Dykman, "The Spectrum of an Oscillator with Fluctuating Mass and Nanomechanical Mass Sensing," Bulletin of the American Physical Society March Meeting, Portland, 15-19 March 2010.

[21] J. Luczka, P. Hanggi and A. Gadomski, "Diffusion of Clusters with Randomly Growing Masses," Physical Review E, Vol. 51, No. 6, 1995, pp. 5762-5769. doi:10.1103/PhysRevE.51.5762

[22] M. S. Abdalla, "Time-Dependent Harmonic Oscillator with Variable Mass under the Action of a Driving Force," Physical Review A, Vol. 34, No. 6, 1986, pp. 4598-4605. doi:10.1103/PhysRevA.34.4598

[23] R. Lambiotte and M. Ausloos, "Brownian Particle Having a Fluctuating Mass," Physical Review E, Vol. 73, No. 1, 2005, Article ID: 011105.

[24] A. Gadomski and J. Siódmiak, "A Kinetic Model of Protein Crystal Growth in Mass Convection Regime" Crystal Research and Technology, Vol. 37, No. 2-3, 2002, pp. 281-291. doi:10.1002/1521-4079(200202)37:2/3<281::AID-CRAT $281>3.3 . \mathrm{CO} ; 2-4$

[25] M. Rub and A. Gadomski, "Nonequilibrium Thermodynamics versus Model Grain Growth: Derivation and Some Physical Implication," Physica A, Vol. 326, No. 3-4, 2003, pp. 333-343. doi:10.1016/S0378-4371(03)00282-6

[26] A. Gadomski, J. Siódmiak, I. Santamara-Holek, J. M.
Rub and M. Ausloos, "Kinetics of Growth Process Controlled by Mass-Convective Fluctuations and Finite-Size Curvature Effects," Acta Physica Polonica B, Vol. 36, No. 5, 2005, pp. 1537-1559.

[27] A. T. Pérez, D. Saville and C. Soria, "Modeling the Electrophoretic Deposition of Colloidal Particles," Europhysics Letters, Vol. 55, No. 3, 2001, pp. 425-431. doi:10.1209/epl/i2001-00431-5

[28] I. Goldhirsch and G. Zanetti, "Clustering Instability in Dissipative Gases," Physical Review Letters, Vol. 70, No. 11, 1993, pp. 1619-1622. doi:10.1103/PhysRevLett.70.1619

[29] S. Luding and H. J. Herrmann, "Cluster-Growth in Freely Cooling Granular Media," Chaos, Vol. 9, No. 3, 1999, pp. 673-682. doi:10.1063/1.166441

[30] I. Temizer, "A Computational Model for Aggregation in a Class of Granual Materials," Master Thesis, University of California, Berkeley, 2001.

[31] W. Benz, "From Dust to Planets," Spatium, Vol. 6, 2000, pp. 3-15.

[32] J. Blum, et al., "Growth and Form of Planetary Seedlings: Results from a Microgravity Aggregation Experiment," Physical Review Letters, Vol. 85, No. 12, 2000, pp. 24262429.

[33] J. Blum and G. Wurm, "Experiments on Sticking, Restructuring and Fragmentation of Preplanetary Dust Aggregates," Icarus, Vol. 143, No. 1, 2000, pp. 138-146. doi:10.1006/icar.1999.6234

[34] S. J. Weidenschilling, D. Spaute, D. R. Davis, F. Marzari and K. Ohtsuki, "Accretional Evolution of a Planetesimal Swarm," Icarus, Vol. 128, No. 2, 1997, pp. 429-455. doi:10.1006/icar.1997.5747

[35] N. Kaiser, "Review of the Fundamentals of Thin-Film Growth," Applied Optics, Vol. 41, No. 16, 2002, pp. 3053-3060. doi:10.1364/AO.41.003053

[36] T. Nagatani, "Kinetics of Clustering and Acceleration in 1D Traffic Flow," Journal of Physical Society Japan, Vol. 65, 1996, pp. 3386-3389. doi:10.1143/JPSJ.65.3386

[37] E. Ben-Naim, P. L. Krapivsky and S. Redner, "Kinetics of Clustering in Traffic Flows," Physical Review E, Vol. 50, No. 2, 1994, pp. 822-829. doi:10.1103/PhysRevE.50.822

[38] M. Ausloos and K. Ivanova, "Mechanistic Approach to Generalized Technical Analysis of Share Prices and Stock Market Indices," European Journal of Physics B, Vol. 27, No. 4, 2002, pp. 177-187. doi:10.1007/s10051-002-9018-9

[39] M. Ausloos and K. Ivanova, "Generalized Technical Analysis. Effects of Transaction Volume and Risk," In: H. Takayasu, Ed., The Applications of Econophysics, Springer Verlag, Berlin, 2004, pp. 117-124.

[40] V. E. Shapiro and V. M. Loginov, "Formulae of Differentiation" and Their Use for Solving Stochastic Equations," Physica A, Vol. 91, 1978, pp. 563-574.

[41] M. Gitterman, "Classical Harmonic Oscillator with Multiplicative Noise," Physica A, Vol. 352, No. 2-4, 2005, pp. 309-334. doi:10.1016/j.physa.2005.01.008 
[42] L. Zhang, S. C. Zhong, H. Peng and M. K. Luo, "Stochastic Multi-Resonance in a Linear System Driven by Multiplicative Polynomial Dichotomous Noise," Chinese Physics Letters, Vol. 28, No. 9. 2011, Article ID: 090505.

[43] M. Gitterman "Order and Chaos: Are They Contradictory or Complementary?" European Journal of Physics, Vol. 23, No. 2, 2002, pp. 119-122. doi:10.1088/0143-0807/23/2/304

[44] M. I. Dykman, R. Mannela, P. V. E. McClintock, F. Moss, and M. Soskin, "Spectral Density of Fluctuations of a Double-Well Duffing Oscillator Driven by White Noise," Physical Review A, Vol. 37, No. 4, 1988, pp. 1303-1313. doi:10.1103/PhysRevA.37.1303

[45] A. Fulinski, "Relaxation, Noise-Induced Transitions, and Stochastic Resonance Driven by Non-Markovian Dichotomic Noise," Physical Review E, Vol. 52, No. 4, 1995, pp. 4523-4526. doi:10.1103/PhysRevE.52.4523

[46] V. Berdichevsky and M. Gitterman, "Multiplicative Stochastic Resonance in Linear Systems: Analytical Solution," Europhysical Letters, Vol. 36, No. 3, 1996, pp. 161-166. doi:10.1209/epl/i1996-00203-9

[47] R. Benzi, S. Sutera and A. Vulpani, "The Mechanism of Stochastic Resonance," Journal of Physics A, Vol. 14, No. 11, 1981, pp. 453-458. doi:10.1088/0305-4470/14/11/006

[48] G. Nicolis, "Stochastic Aspects of Climatic TransitionsResponse to a Periodic Forcing," Tellus, Vol. 34, No. 1, 1982, pp. 1-9. doi:10.1111/j.2153-3490.1982.tb01786.x

[49] L. Gammaitoni, P. Hanggi, P. Jung and F. Marchesoni, "Stochastic Resonance," Review of Modern Physics, Vol. 70, No. 1, 1998, pp. 223-287. doi:10.1103/RevModPhys.70.223

[50] N. G. Stokes, N. D. Stein and V. P. E. McClintock, "Stochastic Resonance in Monostable Systems," Journal of Physics A, Vol. 26, No. 7, 1993, pp. 385-390. doi:10.1088/0305-4470/26/7/007

[51] F. Marchesoni, "Comment on Stochastic Resonance in Washboard Potentials," Physics Letters A, Vol. 352, No. 1-2, 1997, pp. 61-64. doi:10.1016/S0375-9601(97)00232-6

[52] M. Gitterman, "Classical Harmonic Oscillator with Multiplicative Noise," Physica A, Vol. 352, No. 2-4, 2005, pp. 309-334. doi:10.1016/j.physa.2005.01.008

[53] S.-Q. Jiang, B. Wu and T.-X. Gu, "Stochastic Resonance in a Harmonic Oscillator Fluctuating Intrinsic Frequency by Asymmetric Dichotomous Noise," Journal of Electronic Science and Technology, Vol. 5, No. 4, 2007, pp. 344-347.

[54] S. Jiang, F. Guo, Y. Zhow and T. Gu, "Stochastic Resonance in a Harmonic Oscillator with Randomizing Damping by Asymmetric Dichotomous Noise," International
Conference on Communications, Circuits and Systems, Kokura, 11-13 July 2007, pp. 1044-1047.

[55] P. S. Landa and P. V. E. McClintock, "Vibrational Resonance," Journal of Physics A, Vol. 33, No. 45, 2000, pp. 433-438. doi:10.1088/0305-4470/33/45/103

[56] Y. Braiman and I. Goldhirsch, "Taming Chaotic Dynamics With Weak Periodic Perturbations," Physical Review Letters, Vol. 66, No. 20, 1991, pp. 2545-2548. doi:10.1103/PhysRevLett.66.2545

[57] Y. Kim, S. Y. Lee and S. Y. Kim, "Experimental Observation of Dynamic Stabilization in a Double-Well Duffing Oscillator," Physics Letters A, Vol. 275, No. 4, 2000, pp. 254-259. doi:10.1016/S0375-9601(00)00572-7

[58] S. Rajasekar, S. Jeyakumari and M. A. F. Sanjuan, "Role of Depth and Location of Minima of a Double-Well Potential on Vibrational Resonance," Journal of Physics A, Vol. 43, No. 46, 2010, Article ID: 465101.

[59] I. Blekhmam and P. S. Landa, "Conjugate Resonances and Bifurcations in Nonlinear Systems under Biharmonical Excitation," International Journal of Non-Linear Mechanics, Vol. 39, No. 3, 2004, pp. 421-426. doi:10.1016/S0020-7462(02)00201-9

[60] J. P. Baltanas, et al., "Experimental Evidence, Numerics, and Theory of Vibrational Resonance in Bistable Systems," Physical Review E, Vol. 67, No. 6, 2003, Article ID: 066119.

[61] V. N. Chizhevsky and G. Giacomelli, "Improvement of Signal-to-Noise Ratio in a Bistable Optical System: Comparison between Vibrational and Stochastic Resonance," Physical Review A, Vol. 71, No. 1, 2005, Article ID: 011801 .

[62] S. Jeyakumari, V. Chinnathambi, S. Rajasekar and M. A. F. Sanjuan, "Single and Multiple Vibrational Resonance in a Quintic Oscillator with Monostable Potentials," Physical Review E, Vol. 80, No. 4, 2009, Article ID: 046608.

[63] S. Jeyakumari, V. Chinnathambi, S. Rajasekar and M. A. F. Sanjuan, "Analysis of Vibrational Resonance in a Quintic Oscillator," Chaos, Vol. 19, No. 4, 2009, Article ID: 043128 .

[64] J. C. Chedjou, H. B. Fotsin and P. Woafo, "Behavior of the Van der Pol Oscillator with Two External Periodic Forces," Physica Scripta, Vol. 55, No. 4, 1997, pp. 390393. doi:10.1088/0031-8949/55/4/002

[65] H. G. Schuster and W. Just, "Deterministic Chaos: An Introduction," Wiley, New York, 2005. doi: $10.1002 / 3527604804$

[66] E. Ott, "Chaos in Dynamical Systems," Cambridge University Press, Cambridge, 2002. 